\title{
Dynamic characteristics of flow induced vibration in a rotor-seal system
}

\author{
Nan Zhang, Ying Liu and Fulei Chu* \\ Department of Precision Instruments and Mechanology, Tsinghua University, Beijing, P.R. China
}

Received 10 February 2010

Revised 1 July 2010

\begin{abstract}
Flow induced vibration is an important factor affecting the performance of the rotor-seal system. From the point of view of flow induced vibration, the nonlinear models of the rotor-seal system are presented for the analysis of the fluid force, which is induced by the interaction between the unstable fluid flow in the seal and the vibrating rotor. The nonlinear characteristics of flow induced vibration in the rotor-seal system are analyzed, and the nonlinear phenomena in the unbalanced rotor-seal system are investigated using the nonlinear models. Various nonlinear phenomena of flow induced vibration in the rotor-seal system, such as synchronization phenomenon and amplitude mutation, are reproduced.
\end{abstract}

Keywords: Flow induced vibration, rotor-seal system, synchronization phenomenon, amplitude mutation

\section{Introduction}

Flow induced vibration has been a serious problem in many engineering fields, such as wind induced vibration in the high buildings and air elastic vibration in the aircraft, and the fluid elastic vibration of the rotor-seal system in the steam turbine and the hydraulic turbine also belongs to flow induced vibration. Performances of the rotor-seal system are significantly influenced by the dynamic characteristics of flow induced vibration. Flow induced vibration is usually explained as the interaction between the unstable fluid flow in the seal and the vibrating rotor $[1,2]$. The fluid excitation in the seal gap is some forms of the fluid elastic coupling occurred in rotor dynamics, and is also a kind of flow-induced vibration. From the point of view of the fluid elastic coupling, the high rotating speed and harmonic motion of the rotor can result in the fluid to have pulsating flow and vortex shedding, therefore the unstable flow in the seal cavity can be formed. The unstable flow in the seal cavity can produce an excitation on the vibrating rotor and aggravate the rotor vibration in the seal-rotor system. When the pulsating frequency of the fluid in the seal cavity is close to the definite range of the rotor natural frequency, the large amplitude of vibration of the rotor can be formed and the fluid excitation in the seal gap will be induced, which significantly affects the safe operation of the rotor system. It will be very helpful to understand the nonlinear behaviors of flow induced vibration in the design of the system. With the increase of the rotating speed, investigation on the dynamic characteristics of flow induced vibration has become one of the key issues in the rotor-seal system.

Many models of the rotor-seal system, such as the conventional model with eight parameters and the Muszynska's model, have been investigated and can be found in many references. For the previous research, it had been assumed that the interaction between fluid and rotor was coupled together by springs and dampers, and that the rotor was only with a small displacement, and the fluid force in the rotor-seal system was expressed as a linear function. The dynamic coefficients of the rotor-seal system have been studied by linearized fluid forces [3]. Hua [4] established the models of the rotor-seal system by using Muszynska's seal forces. The Hopf bifurcation behaviors of a symmetric

\footnotetext{
${ }^{*}$ Corresponding author. E-mail: chufl@mail.tsinghua.edu.cn.
} 

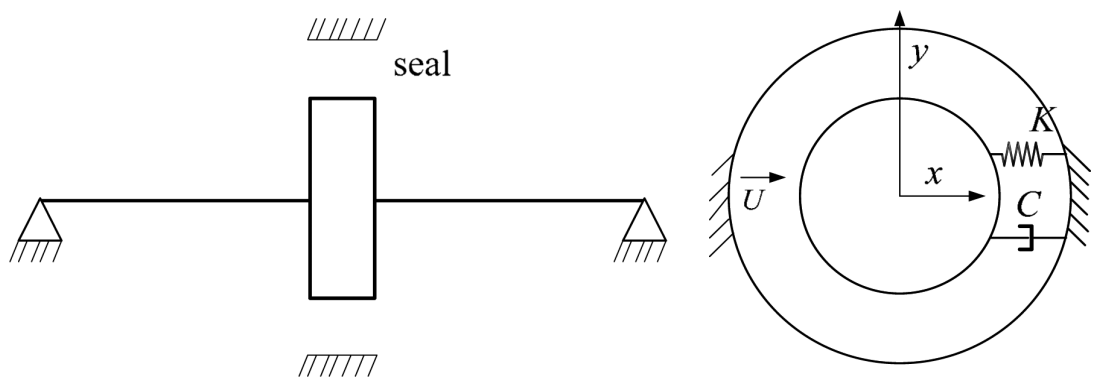

Fig. 1. Rotor-seal system.

rotor-seal system were investigated using Muszynska's dynamic force model of the seal fluid [5]. Cheng [6] had analyzed the complicated non-linear vibrations of a rotor-bearing-seal system using a model considering non-linear oil-film forces and Muszynska's seal forces. The conventional model with eight parameters was developed by Muszynska's model at certain extent. It is no doubt that Muszynska's model is a very good model for the analysis of the rotor-seal system, but it is of very limited to describe the rotor motion of the large amplitude and fluid excitation in the seal gap in the rotor-seal system. Firstly, the premise of the linear model is small vibration, but when the fluid excitation in the seal gap occurs, the rotor will be with the vibration of the large amplitude. Therefore the linear model is not perfect enough. Secondly, the fluid excitation in the seal gap is self-excited vibration, and with the nonlinear nature. If the linear model was used to describe the rotor motion, the rotor-seal system would lose the most essential dynamic phenomena. This linear model cannot describe the nonlinear characteristics, such as the amplitude of mutations, the frequency synchronization and so on.

Generally the investigations on the fluid force are concerned with the computation of the Navier-Stokes equation. For example, the fluid flow force through the labyrinth seal was obtained by employing computational fluid dynamics based on the Navier-Stokes equation [7]. A numerical procedure for computing the Navier-Stokes equation was presented in the rotor-seal system [8]. He [9] studied gas flow-induced vibration in a three-dimensional rotor seal system using Navier-Stokes equation. It is highly effective solution for computing the Navier-Stokes equation to solve the fluid force on the vibrating rotor. But it will suffer from great difficulty when using this method in the selection of the used parameters and solving equation on the theory.

In this paper, based on flow induced vibration theory, the nonlinear vibration models are established to describe the rotor-seal system, and the dynamic characteristics of flow induced vibration in the rotor-seal system are investigated.

\section{Mathematical model}

From the point of view of flow induced vibration, the question of the flow vibration in the system with the fluid flow in the seal and the vibrating rotor is investigated. When the rotor runs fast, the pulsating flow and the unstable fluid flow are generated through the interaction between the rotor and the fluid. Then flow induced vibration of the system is produced by the unstable fluid flow. The pulsating flow induced vibration will be investigated on a single disk rotor-seal system. The model is shown in Fig. 1, and the differential equation of the rotor motion under the action of the fluid excitation in the seal gap is defined as $[10,11]$

$$
m \frac{d^{2} x}{d t^{2}}+C \frac{d x}{d t}+K x=F
$$

In this equation, $x$ is the vibration displacement of the disk centre, and $m$ is the mass of the disk, $C$ and $K$ represent damping and stiffness coefficients respectively, $F$ is the fluid force assumed to be acting on the disk of the shaft. In engineering, the analysis of the fluid force $F$ on the flow direction in the pulsating flow induced vibration is obtained using the Morison method [10]. This fluid force $F$ is composed of two components, the inertial force $F_{l}$ and the pull $F_{D}$. The inertial force is produced by the operated flow acceleration, and the pull is in-phase with the flow velocity. Because the additional mass among inertial force is directly proportional to the relative acceleration between the 
disk and the fluid, the accelerated disk in the rotor-seal system is exerted by the inertial force which can be defined as [10]

$$
F_{l}=\rho A L \frac{d U}{d t}+C_{l} \rho A L\left(\frac{d U}{d t}-\frac{d^{2} x}{d t^{2}}\right)
$$

where $\rho$ is the fluid density, $U$ is the flow velocity, $A$ represents the section area of the disk, $C_{l}$ is the additional mass coefficient, $L$ is the width of the disk, $\frac{d U}{d t}$ is the fluid acceleration, $\frac{d^{2} x}{d t^{2}}$ is the disk acceleration, and $\frac{d U}{d t}-\frac{d^{2} x}{d t^{2}}$ is the relative acceleration between the disk and the fluid. The pull on the disk is defined as [10]

$$
F_{D}=0.5 \rho\left|U-\frac{d x}{d t}\right|\left(U-\frac{d x}{d t}\right) D L C_{D}
$$

where $D$ is the diameter of the disk, and $C_{D}$ is the dynamic coefficient. The orientation of the pull is along with the relative speed $\left(U-\frac{d x}{d t}\right)$ between the disk and the fluid. Therefore this fluid force can be determined by

$$
F=F_{l}+F_{D}=\rho A L \frac{d U}{d t}+C_{l} \rho A L\left(\frac{d U}{d t}-\frac{d^{2} x}{d t^{2}}\right)+0.5 \rho\left|U-\frac{d x}{d t}\right|\left(U-\frac{d x}{d t}\right) D L C_{D}
$$

Based on Eq. (4), Eq. (1) can be re-written as

$$
M \frac{d^{2} x}{d t^{2}}+C \frac{d x}{d t}+K x=\rho A L C_{m} \frac{d U}{d t}+0.5 \rho\left|U-\frac{d x}{d t}\right|\left(U-\frac{d x}{d t}\right) D L C_{D}
$$

In Eq. (5), $M=m+C_{l} \rho A L$ and represents the overall mass of the disk and the fluid, $C_{m}$ is the inertial coefficient and can be written as $C_{m}=1+C_{l}$.

The flow velocity is far more than the vibration velocity of the system in the rotor-seal system, namely, $U>>\frac{d x}{d t}$, therefore the Eq. (5) can be simplified as

$$
M \frac{d^{2} x}{d t^{2}}+C \frac{d x}{d t}+K x=\rho A L C_{m} \frac{d U}{d t}+0.5 \rho|U| U D L C_{D}
$$

\section{Theoretical analysis and computation}

\subsection{For pulsating flow induced vibration}

Dynamic coefficient is connected with Reynolds number and usually a function of the Reynolds number. Dynamic coefficient $C_{D}$ in the theory is close to 0 when Reynolds number is extremely big. Dynamic coefficient $C_{D}$ is generally between 1 and 2 when Reynolds number is 100. If the dynamic coefficient $C_{D}$ and the inertial coefficient $C_{m}$ did not change along with time, the vibration characteristics of the rotor-seal system under the action of pulsating flow induced vibration would be analyzed in the following.

a). When the flow velocity is an average value among the fluid field, namely $U=U_{0}$, where $U_{0}$ is a constant, Eq. (6) is written as

$$
M \frac{d^{2} x}{d t^{2}}+C \frac{d x}{d t}+K x=\frac{1}{2} \rho U_{0}^{2} D L C_{D}
$$

b). When the flow velocity is in a form of sine wave (namely $U=U_{m} \sin \omega t$, where $U_{m}$ is the amplitude of the flow velocity, $\omega$ is the pulsating frequency of the fluid), $|U| U$ in Eq. (6) is expanded into a Fourier series, namely $|U| U=U_{m}^{2} \sum_{n=1,3,5}^{\infty} C_{n} \sin n \omega t$, where $C_{n}=2\left(1-\frac{n^{2}}{n^{2}-4}\right) / n \pi$. Then Eq. (6) can be written as

$$
M \frac{d^{2} x}{d t^{2}}+C \frac{d x}{d t}+K x=\rho A L C_{m} U_{m} \omega \cos \omega t+\frac{1}{2} \rho U_{m}^{2} D L C_{D} \sum_{n=1,3,5}^{\infty} C_{n} \sin n \omega t
$$

It is shown from Eq. (8) that the operating frequency of the rotor is the pulsating frequency $\omega$ of the fluid. The vibration component $x$ can also be expressed in a Fourier series as 


$$
x=\sum_{n=1}^{\infty}\left(a_{n} \sin n \omega t+b_{n} \cos n \omega t\right)
$$

where $a_{n}$ and $b_{n}$ are Fourier coefficients in Eq. (9). Based on Eqs (8) and (9), $a_{n}$ and $b_{n}$ can be determined, and the solution of vibration displacement $x$ can be written as

$$
\begin{aligned}
x= & C_{m} \frac{\omega}{\omega_{n}} \frac{\rho A L}{M} \frac{U_{m}}{\omega_{n}}\left\{\left[1-\left(\frac{\omega}{\omega_{n}}\right)^{2}\right] \cos \omega t+2 \varepsilon \frac{\omega}{\omega_{n}} \sin \omega t\right\} M_{1} \\
& +0.5 C_{D} \frac{\rho D L}{M}\left(\frac{U_{m}}{\omega_{n}}\right)^{2} \sum_{n=1,3,5}^{\infty} C_{n} \cdot\left\{\left[1-\left(\frac{n \omega}{\omega_{n}}\right)^{2}\right] \sin n \omega t+2 \varepsilon n \frac{\omega}{\omega_{n}} \cos n \omega t\right\} M_{n}
\end{aligned}
$$

where $\omega_{n}$ is named as first natural frequency of the rotor and written as $\omega_{n}=\sqrt{\frac{K}{M}}, \varepsilon$ is the damping ratio and written as $\varepsilon=\frac{C}{2 M \omega_{n}}, M_{n}$ is the magnification coefficient in the system and is written as

$$
M_{n}=\left\{\left[1-\left(\frac{n \omega}{\omega_{n}}\right)^{2}\right]^{2}+\left(2 \varepsilon \frac{n \omega}{\omega_{n}}\right)^{2}\right\}^{-1}
$$

When the operating frequency $\omega$ is close to the first natural frequency of the rotor $\omega_{n}$ or close to $\frac{1}{3} \omega_{n}$, $\frac{1}{5} \omega_{n} \ldots \ldots$, resonance phenomena would occur in the rotor-seal system. Moreover, at the time of the resonance occurrence, the response characteristic (see Eq. (10)) of the rotor-seal system is related with damping ratio $\varepsilon$. When $\omega$ is close to $\omega_{n}$, resonance phenomena occur and the maximal amplitude of displacement $x$ also occurs at the moment.

c). When the flow velocity is in the form of the sum of a sine wave and a constant, namely $U=U_{0}+U_{m} \sin \omega t$, where $U_{0}$ and $U_{m}$ are the bigger than zero, and when $U_{0}>U_{m}$, Eq. (6) is written as

$$
\begin{aligned}
& M \frac{d^{2} x}{d t^{2}}+C \frac{d x}{d t}+K x=\rho A L C_{m} U_{m} \omega \cos \omega t \\
& +\frac{1}{2} \rho D L C_{D}\left(U_{0}^{2}+0.5 U_{m}^{2}+2 U_{0} U_{m} \sin \omega t-0.5 U_{m}^{2} \cos 2 \omega t\right)
\end{aligned}
$$

The solution of vibration displacement $x$ can be expressed as

$$
\begin{aligned}
x= & \frac{1}{2} C_{D} \frac{\rho D L}{M}\left[\frac{U_{0}^{2}+0.5 U_{m}^{2}}{\omega_{n}^{2}}\right]+C_{m} \frac{\omega}{\omega_{n}} \frac{\rho A L}{M} \frac{U_{m}}{\omega_{n}}\left\{\left[1-\left(\frac{\omega}{\omega_{n}}\right)^{2}\right] \cos \omega t+2 \varepsilon \frac{\omega}{\omega_{n}} \sin \omega t\right\} M_{1} \\
& +C_{D} \frac{\rho D L}{M}\left(\frac{U_{0} U_{m}}{\omega_{n}^{2}}\right)\left\{\left[1-\left(\frac{\omega}{\omega_{n}}\right)^{2}\right] \sin \omega t+2 \varepsilon \frac{\omega}{\omega_{n}} \cos \omega t\right\} M_{1} \\
& -\frac{1}{4} C_{D} \frac{\rho D^{2} L}{M}\left(\frac{U_{m}}{\omega_{n}}\right)\left\{\left[1-\left(\frac{2 \omega}{\omega_{n}}\right)^{2}\right] \sin 2 \omega t+4 \varepsilon \frac{\omega}{\omega_{n}} \cos 2 \omega t\right\} M_{2}
\end{aligned}
$$

where the response characteristics of the rotor can be obtained from Eq. (13), and the static displacement is induced because of the constant $U_{0}$. The resonance phenomena would occur when the operating frequency $\omega$ of the rotor (or the pulsating frequency $\omega$ of the fluid) is close to the first natural frequency $\omega_{n}$ of the rotor or close to $0.5 \omega_{n}$.

\subsection{For vortex shedding induced vibration}

When the fluid with the average velocity along the vertical rotor direction passes through the rotor, the vortex shedding in the seal cavity will be formed when the Reynolds number $\in\left[10^{3} \sim 10^{5}\right]$. A fluid force perpendicular to the flow direction is generated, and the fluid force of the alternating change results in the vortex shedding induced vibration in the rotor-seal system. Vortex shedding induced vibration is a kind of flow induced vibration, and a kind of self-excited vibration. Based on Wake oscillation model [10], the change of the fluid force is with the characteristic of self-excited vibration, and the fluid dynamic coefficient $C_{D}$ is obtained using the equation of self-excited vibration, thus the dynamic coefficient $C_{D}$ can be expressed by the following equation [10] 


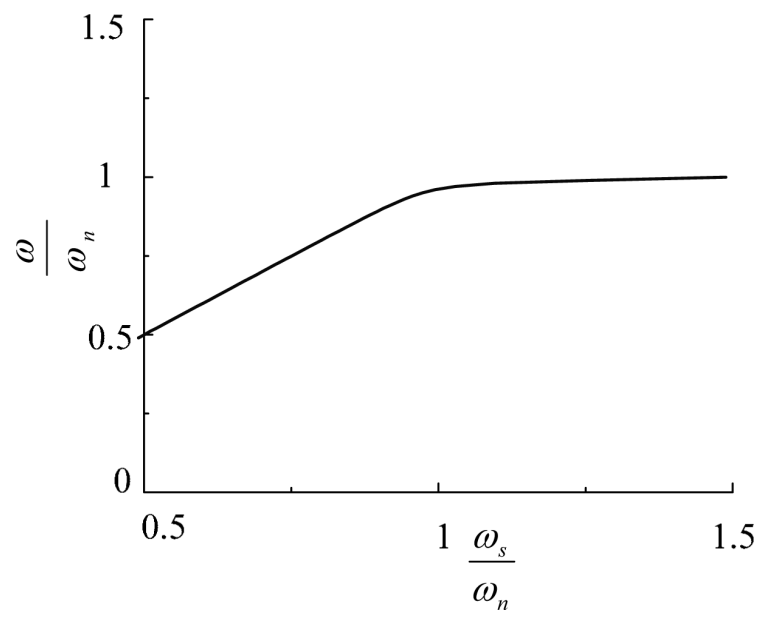

Fig. 2. Frequency capture of flow induced vibration.

$$
\frac{d^{2} C_{D}}{d t^{2}}-\left[a \omega_{s} \frac{d C_{D}}{d t}-\frac{r}{\omega_{s}}\left(\frac{d C_{D}}{d t}\right)^{3}\right]+\omega_{s}^{2} C_{D}=\frac{b \omega_{n}}{D} \frac{d y}{d t}
$$

In this equation, $y$ is the vibration displacement of the disk centre, $\omega_{s}$ is the Strouhal frequency and written as $\omega_{s}=2 \pi S U / D$, where $S$ is Strouhal number of the fluid and a dimensionless constant. $\omega_{s}$ represents main frequency of the variational fluid when the rotor is not running. In addition, $a, r$ and $b$ are all empirical coefficients.

The nonlinear vibration models with vortex shedding induced vibration in the rotor-seal system are the following equations

$$
\begin{aligned}
& \frac{d^{2} y}{d t^{2}}+2 \varepsilon \omega_{n} \frac{d y}{d t}+\omega_{n}^{2} x=\frac{1}{2} \frac{\rho U_{0}^{2} D L}{M} C_{D} \\
& \frac{d^{2} C_{D}}{d t^{2}}-\left[a \omega_{s} \frac{d C_{D}}{d t}-\frac{r}{\omega_{s}}\left(\frac{d C_{D}}{d t}\right)^{3}\right]+\omega_{s}^{2} C_{D}=\frac{b \omega_{n}}{D} \frac{d y}{d t}
\end{aligned}
$$

The model of Eq. (15) is with the characteristic of the fluid-solid coupling interaction, and describes comprehensively the interaction between the unstable fluid flow in the seal and the vibrating rotor. Vibration component $x$ is expressed in a Fourier series as

$$
y=\sum_{n=1}^{\infty}\left(a_{n x} \sin n \omega t+b_{n x} \cos n \omega t\right)
$$

In this equation, $\omega$ is the operating frequency of the rotor and is also the pulsating frequency of the fluid. Based on the harmonic balance method, when $n=1$, the equation of the frequency for Eq. (15) is determined by

$$
\left[\left(\frac{\omega_{s}}{\omega_{n}}\right)^{2}-\left(\frac{\omega}{\omega_{n}}\right)^{2}\right]\left\{\left[1-\left(\frac{\omega}{\omega_{n}}\right)^{2}\right]^{2}+\left(2 \varepsilon \frac{\omega}{\omega_{n}}\right)^{2}\right\}-\frac{\varepsilon b \rho U_{0}^{2} L}{M \omega_{n}^{2}}\left(\frac{\omega}{\omega_{n}}\right)^{2}=0
$$

Based on this equation of the frequency, the operating frequency $\omega$ of the rotor (or the pulsating frequency $\omega$ of the fluid) is affected by system parameters, the first natural frequency $\omega_{n}$ of the rotor and the Strouhal frequency $\omega_{s}$. The vortex induced vibration in the rotor-seal system is also affected by many other factors, and the damping ratio and the rotational speed are all prone to result in the occurrence of the vortex induced vibration. Based on the mathematical model and computation, Fig. 2 is obtained from Eq. (17). As shown in Fig. 2, when the Strouhal frequency $\omega_{s}$ is close to the definite range of the first natural frequency $\omega_{n}$, the Strouhal frequency $\omega_{s}$ and the operating frequency $\omega$ of the rotor are all seized by first natural frequency $\omega_{n}$, namely $\frac{\omega_{s}}{\omega_{n}} \approx 1, \quad \frac{\omega}{\omega_{n}} \approx 1$, and frequency capture is also obtained. Frequency capture is a characteristic of self-excited vibration, and the model of Eq. (15) is the nonlinear vibration system under the action of self-excited vibration, so the computing results of Fig. 2 are consistent with 


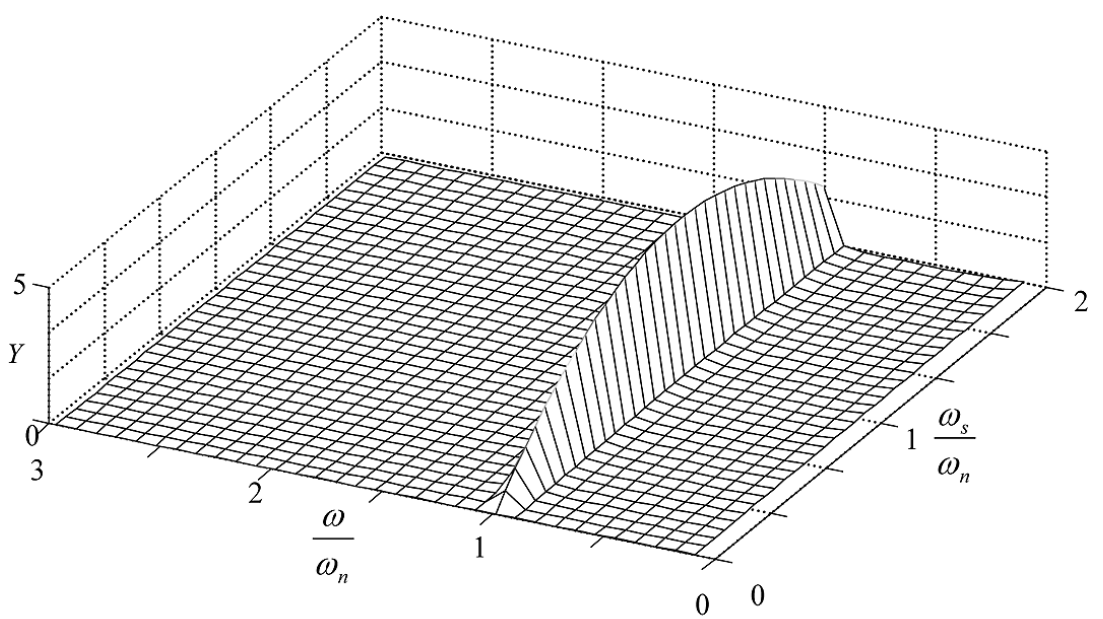

(a) Three-dimensional drawing of amplitude
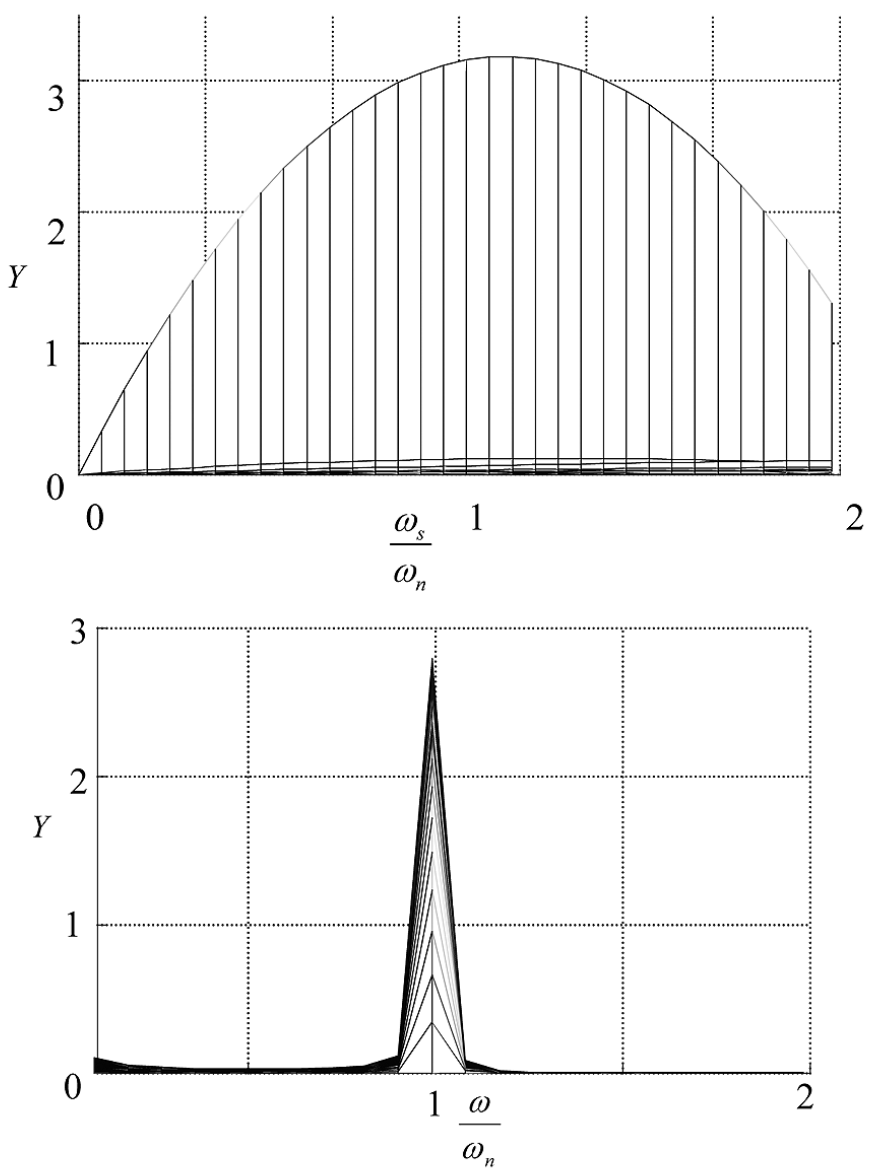

(b) Amplitude plane

Fig. 3. Jumping phenomenon of the amplitude. 
the theory analysis. Frequency capture can be explained that the Strouhal frequency $\omega_{s}$ is seized by $\omega_{n}$ when the Strouhal frequency $\omega_{s}$ is close to the definite range of the first natural frequency $\omega_{n}$, then the Strouhal frequency $\omega_{s}$ do not change with the fluid speed. The phenomena are also named as synchronization phenomena or the locked frequency.

The interaction between the equation of the frequency and the nonlinear vibrating model of the fluid-solid coupling interaction in the rotor-seal system is comprehensively described by the following equation

$$
r\left(\frac{\omega}{\omega_{n}}\right)^{2} C^{2}=\left\{\frac{b A_{1}}{D}\left[1-\left(\frac{\omega}{\omega_{n}}\right)^{2}\right] M_{1}+\frac{a \omega_{s}}{\omega_{n}}\right\} \frac{\omega_{s}}{\omega_{n}}
$$

where $C$ is the amplitude of the dynamic coefficient $C_{D}$. The solution for the amplitude of displacement $y$ is the following equation

$$
Y=\frac{A_{1} C}{\sqrt{\left[1-\left(\frac{\omega}{\omega_{n}}\right)^{2}\right]^{2}+\left(2 \varepsilon \frac{\omega}{\omega_{n}}\right)^{2}}}
$$

In Eq. (19), $Y$ represents the amplitude of displacement $y$ and

$$
A_{1}=\frac{\rho U_{0}^{2} D L}{2 M \omega_{n}^{2}}
$$

Based on Eqs (17) and (19), relation between the amplitude and the frequencies $\omega, \omega_{n}, \omega_{s}$ is shown in Fig. 3. When the Strouhal frequency $\omega_{s}$ of the fluid is close to the definite range of the first natural frequency $\omega_{n}$, synchronization phenomena is obtained. The synchronization phenomena caused by the vortex induced vibration are more severe than by the general resonance. Because the vibration speed of the system affects the amplitude of the excitation, and the amplitude is greatly enhanced. As shown in Fig. 3, the generated amplitude increasing in the vortex induced vibration is named as the phenomenon of the amplitude mutation. Amplitude mutation is one prominent characteristic of flow induced vibration, and also named as jumping phenomenon of the amplitude.

\section{Conclusions}

The nonlinear models of the rotor-seal system are established from the point of view of flow induced vibration. The analysis scheme of rotor self-excited vibration is adopted to investigate the nonlinear behaviors of the rotorseal system. The nonlinear models of the rotor-seal system are described to analyze and compute the dynamic characteristic of flow induced vibration, using the changes of the fluid flow parameters, such as the flow velocity in a form of sine wave or and the constant or a average velocity. The frequency capture and the jumping phenomenon of the amplitude are also found to depict the characteristics of flow induced vibration in the rotor-seal system. The investigation also demonstrates that the proposed method, from the point of view of flow induced vibration, can be effectively applied to the nonlinear analysis of the rotor-seal system.

\section{Acknowledgements}

The authors gratefully acknowledge that the work was supported by Natural Science Foundation of China (NSFC) under the project Nos. 10732060 and 50975157.

\section{References}

[1] W. Wu, J. Yuan and L. Cheng, Multi-high-frequency perturbation effects on flow-induced vibration control, Journal of Sound and Vibration 305 (2007), 226-242. 
[2] D.H. Kim and S.H. Chang, Flow-induced vibration in two-phase flow with wire coil inserts, International Journal of Multiphase Flow 34 (2008), 325-332.

[3] A. Muszynska, Improvements in lightly loaded rotor/bearing and rotor/seal models, Journal of Vibration, Acoustics, Stress and Reliability in Design 110 (1988), 129-136.

[4] J. Hua, S. Swaddiwudhipong, Z.S. Liu and Q.Y. Xu, Numerical analysis of nonlinear rotor-seal system, Journal of Sound and Vibration 283 (2005), 525-542.

[5] Q. Qing, J.E. Cooper and A.Y.T Leung, Hopf bifurcation analysis of a rotor/seal system, Journal of Sound and Vibration 252 (2002), 817-833.

[6] M. Cheng, G. Meng and J.P. Jing, Numerical and experimental study of a rotor-bearing-seal system, Mechanism and Machine Theory 42 (2007), 1043-1057.

[7] W.Z. Wang, Y.Z. Liu and P.N. Jiang, Numerical analysis of leakage flow through two labyrinth seal, Journal of Hydrodynamics 18 (2006), 449-457.

[8] W.B. Duan, F.L. Chu, C.H. Kim and Y.B. Lee, A bulk-flow analysis of static and dynamic characteristics of floating ring seals, Tribology International 40 (2007), 470-478.

[9] L.D. He, J.J. Gao and Y. Jin, Study on gas flow-induced vibration for a three-dimensional rotor-seal system. Chinese Journal of Mechanical Engineering 39 (2009), 100-104 (in Chinese).

[10] R.D. Blevin, Flow-induced vibration, Machinery industry Publishing Housing, Beijing, 1983, pp. 122-133 (in Chinese)

[11] W.J. Ding, Self-excited vibration in the engineering, Changchun Education Press, Changchun, 1988, pp. 227-246 (in Chinese). 

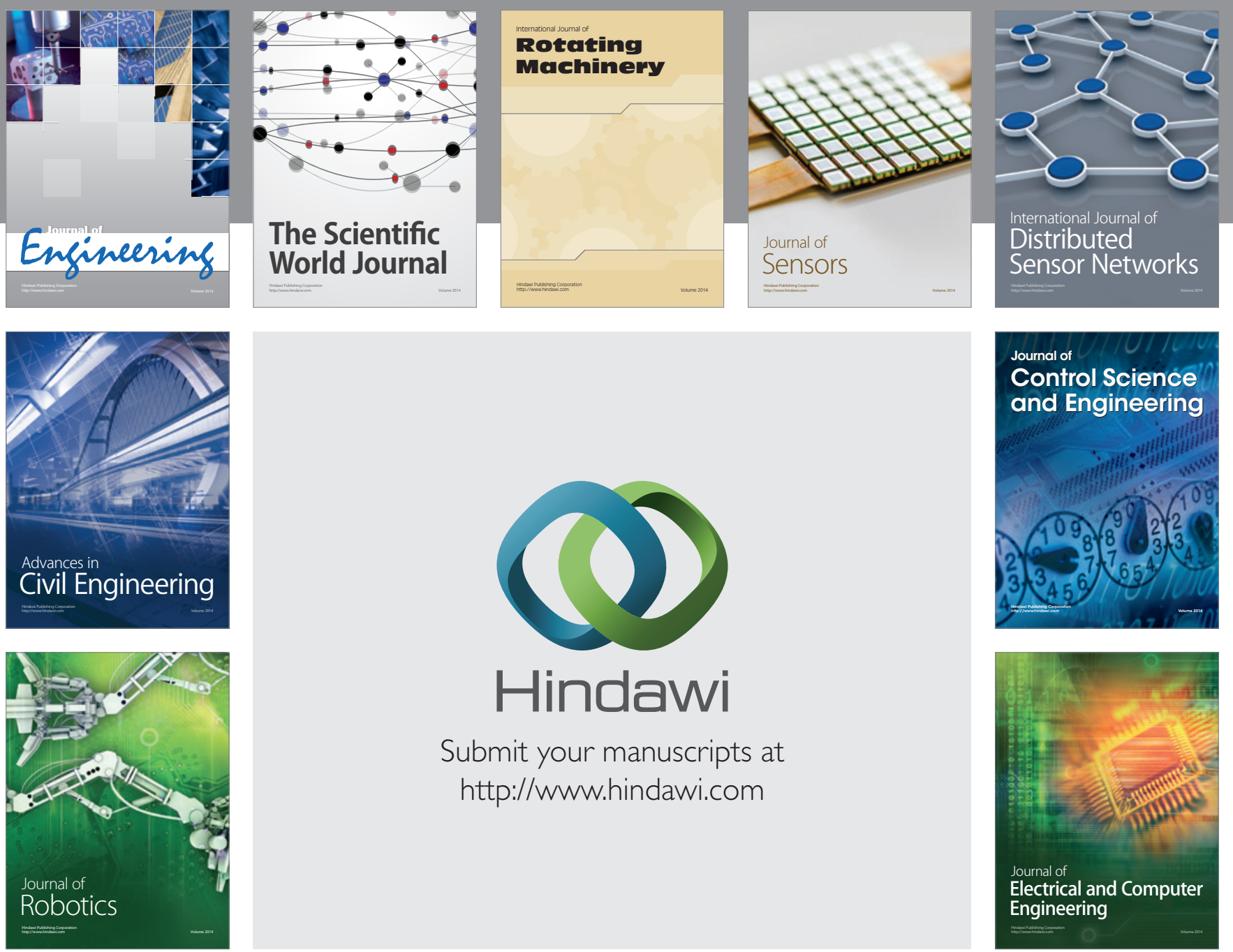

Submit your manuscripts at

http://www.hindawi.com
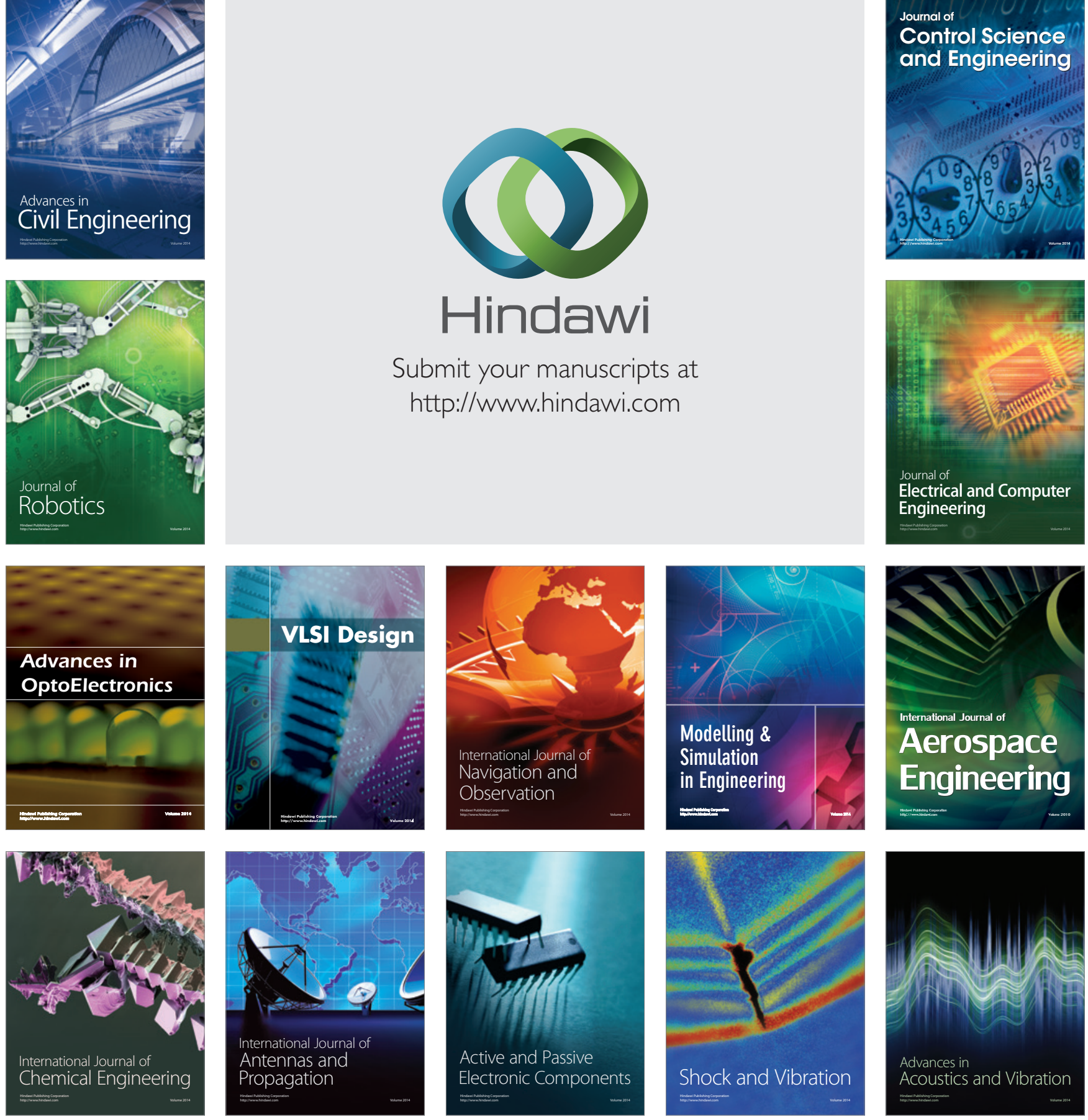